\title{
Ocular masquerade syndrome
}

Keywords: masquarade, syndrome, pseudo-uveitis, ocular, inflammation, neoplasia, tumor, resistance to steroids

Abbrevations: OMS, ocular masquarade syndromes; JXG, juvenile xanthogranuloma; CAR, carcinoma-associated retinopathy; MAR, melanoma-associated retinopathy; PRL, primary retinal lymphoma; CNS, central nervous system; PCNSL, primary CNS lymphoma.

\section{Editorial}

In the ophthalmologic literature, the term "masquerade syndrome" has been firstly used in 1967 for the description of a conjunctival carcinoma presenting as chronic conjunctivitis, an extraocular inflammatory disorder. ${ }^{1}$ However, especially a group of malignant and non-malignant systemic or primary ocular diseases mimicking immune-mediated uveitis have been also included into the spectrum of this syndrome. Ocular Masquerade Syndrome (OMS) is often misdiagnosed as chronic uveitis, in the other words, OMS is a group disease of the causes of pseudo-uveitis. However, in OMS, cells arise from a non-inflammatory origin (intraocular pigment, blood, or neoplastic cells). The most common features of this entity are bilateral asymmetrical involvement, the presence of aqueous and/ or vitreous cells, older age, history of systemic or ocular malignancy, the absence of other inflammatory signs such as ocular pain, keratic precipitates and synechiae, and initial response and eventual resistance to corticosteroids..$^{2-4}$ OMS usually points out malignant entities including sebaceous gland carcinoma (meibomian gland carcinoma or sebaceous adenocarcinoma), intraocular and periocular lymphoma, retinoblastoma, diffuse amelanotic ocular melanoma and metastatic tumors to the choroid and retina. The incidence of malignant entities in OMS is $2-8 \%$ of cases in a uveitis practice..$^{2-4}$

However, some non-malignant or non-neoplastic proliferative conditions can be considered OMS. These include uveal juvenile xanthogranuloma (JXG), fungal or chronic bacterial endophthalmitis (P. Acnes infection, etc), scleritis/episcleritis, intraocular foreign body, siderosis, amyloidosis, pigment dispersion syndrome, old vitreous hemorrhage, retinal vascular occlusions or retinal ischemia, iridocorneo-endothelial syndrome (ICE), retinitis pigmentosa, anterior segment ischemia caused by carotid artery disease or irradiation, ocular ischemic syndrome, and chronic peripheral retinal detachment, paraneoplastic syndromes such as carcinoma-associated retinopathy (CAR)/melanoma-associated retinopathy (MAR), bilateral diffuse uveal melanocytic proliferation caused by intraocular dispersion or migration of cells. ${ }^{2-13}$ The most frequent neoplastic cause is primary intraocular or retinal lymphoma (PRL) having a high-grade neoplasia, an aggressive course, neural retinal infiltration, and central nervous system (CNS) involvement. However, primary CNS lymphoma (PCNSL) that is extranodal, non-Hodgkin's B cell lymphoma involving CNS or eye or both, primary choroidal or iridal lymphoma or secondary intraocular infiltration of a systemic lymphoma may mimic an intraocular inflammatory condition. In the suspicion of a steroid resistant bilateral uveitis in especially patients between 50 and 60 years of age, PRL should be excluded. The most common symptoms of the PRL include painless visual loss and "floaters". The
Volume 8 Issue 3 - 2018

\author{
Burak Turgut \\ Private Etimed Hospital, Ophthalmology Clinic, Turkey
}

Correspondence: Burak Turgut, Private Etimed Hospital, Ophthalmology Clinic, Elvan Mah. 1934. Sok. No:4 Etimesgut / ANKARA, Tel +90 (3/2) 29306 06, Email drburakturgut@gmail.com

Received: May 10, 2018 | Published: May 14, 2018

most common signs are anterior chamber involvement (over 30\%), multiple yellow-orange subretinal pigment epithelial lesions (leopard skin appearance on fluorescein angiography).

In contrast, primary choroidal lymphoma (PCL), B cell lymphoma of mucosa-associated lymphoid tissue (MALT) type has a low-grade and a good prognosis. The majority of primary iridal lymphomas are high-grade neoplasia, but relatively good prognosis. ${ }^{2-5,8,9}$ The main causes of OMS in adults are PRL, primary intraocular lymphoma, ocular metastasis of systemic Non-Hodgkin lymphoma, or breast/ lung/renal carcinomas, or uveal melanomas. In children, OMS usually occurs due to leukemia, retinoblastoma, medulloepithelioma or juvenile xanthogranuloma or persistent hyperplastic primary vitreous. In malignant causes, isolated chorioretinal lesions, serous retinal detachment, and iris infiltration can seem while as in nonmalignant conditions, chorioretinal lesions with surrounding cellular infiltrate and pseudo-retinal vasculitis may be observed. ${ }^{2-13}$ Lymphoma, leukemia, sebaceous gland carcinoma and retinoblastoma are a life-threatening diseases, and OMS may be the first presentation of these diseases. Almost a half of the cases with OMS has an underlying malignant disease. $^{2-13}$ The importance of OMS arises from the possibility of life rescue with the early recognition and prompt treatment of underlying malignancy. Thus, in each case with non-responsiveness to treatment with corticosteroids for possible uveitis, OMS should be considered.

\section{Acknowledgments}

None.

\section{Conflict-of-interest}

The author declares that there is no conflict of interest regarding the publication of this paper.

\section{Authorship contributions}

Concept and design, data collection, literature Search, writing: analysis, interpretation: burak turgut.

\section{References}

1. Theodore FH. Conjunctival carcinoma masquerading as chronic conjunctivitis. Eye Ear Nose Throat Mon. 1967;46(11):1419-1420. 
2. Tsai T, O’Brien JM. Masquerade syndromes: malignancies mimicking inflammation in the eye. Int Ophthalmol Clin. 2002;42(1):115-131.

3. Peng MY, Kersten RC. The Masquerade Syndrome. JAMA Ophthalmol. 2017;135(2):161-162.

4. Coupland SE. Neoplastic masquerade syndromes. Essentials in Ophthalmology Uveitis and Immunological Disorders. In: Pleyer U, Foster CS, editors. Verlag Berlin, Heidelberg, Springer; 2007. p. 157-175.

5. Read RW, Zamir E, Rao GN. Neoplastic masquerade syndromes. Surv Ophthalmol. 2002;47(2):81-124.

6. Dadeya S, Malik KP, Guliani BP, et al. Acute lymphocytic leukemia presenting as masquerade syndrome. Ophthalmic Surg Lasers. 2002;33(2):163-165.

7. Rosenthal AR. Ocular manifestations of leukemia-a review Ophthalmology. 1983;90(8):899-905.

8. Abe RY, Pinto RD, Bonfitto JF, et al. Ocular masquerade syndrome associated with extranodal nasal natural killer/T-cell lymphoma: case report. Arq Bras Oftalmol. 2012;75(6):430-432.
9. Cassoux N, Merle-Beral H, Leblond V, et al. Ocular and central nervous system lymphoma: clinical features and diagnosis. Ocul Immunol Inflamm. 2000;8(4):243-250.

10. Makhoul D, Kolyvras N, Benchekroun S, et al. Sickle cell crisis presenting as a masquerade syndrome complicated by macular ischemia. Ocul Immunol Inflamm. 2010;18(3):178-180.

11. Rothova A, Ooijman F, Kerkhoff F, et al. Uveitis masquerade syndromes. Ophthalmology. 2001;108(2):386-399.

12. Kubicka-Trzaska A, Romanowska-Dixon B. Malignant uveitis masquerade syndromes. Klin Oczna. 2008;110(4-6):199-202.

13. Kubicka-Trzaska A, Romanowska-Dixon B. Non-malignant uveitis masquerade syndromes. Klin Oczna. 2008;110(4-6):203-206. 